\title{
Diagnostic Accuracy of Strain Imaging of the Left Ventricle in Detection of Obstructive Coronary Artery Disease
}

\author{
Mohamed Fahmy Elnoamany ${ }^{1 *}$, Waleed Abdou Ibrahim¹, Ahmed Mahmoud Hamza ${ }^{2 *}$ \\ ${ }^{1}$ Menoufia University, Menoufia, Egypt \\ ${ }^{2}$ Mahalla Cardiac Center, Gharbia, Egypt \\ Email: ^mnoamany@hotmail.com, ^Ahmedhamza949@gmail.com
}

How to cite this paper: Elnoamany, M.F., Ibrahim, W.A. and Hamza, A.M. (2020) Diagnostic Accuracy of Strain Imaging of the Left Ventricle in Detection of Obstructive Coronary Artery Disease. World Journal of Cardiovascular Diseases, 10, 572-586. https://doi.org/10.4236/wjcd.2020.108057

Received: April 30, 2020

Accepted: August 28, 2020

Published: August 31, 2020

Copyright $\odot 2020$ by author(s) and Scientific Research Publishing Inc. This work is licensed under the Creative Commons Attribution International License (CC BY 4.0).

http://creativecommons.org/licenses/by/4.0/

\begin{abstract}
Background: Speckle tracking echocardiography using average global strain and strain rate (SR) parameters for early detection of high risk patients with coronary artery disease (CAD) has gained a substantial clinical interest. Objective: Assessment of the diagnostic accuracy of strain imaging of the left ventricle in detection of obstructive coronary artery disease. Methods: One hundred patients were enrolled. They were divided into 3 groups: Group I (n $=40$ patients) presented with ST segment elevation myocardial infarction (STEMI), Group II ( $\mathrm{n}=40$ patients) presented with non-ST segment elevation myocardial infarction (NSTEMI) or unstable angina (UA) and Group III ( $\mathrm{n}=20$ patients) with normal coronary angiography served as a control group. All patients were subjected to $2 \mathrm{D}$ speckle tracking echocardiography (2D STE) to assess LV longitudinal strain and strain rate (SR). Sensitivity, specificity and diagnostic accuracy of 2D STE in prediction of CAD and its severity using Gensini score were assessed. Results: The mean age for Group I, II and III was $52.20 \pm 11.83,51.97 \pm 14.53$ and $52.75 \pm 10.75$ respectively. LV average global systolic strain (AGS) was significantly lower in group I and II when compared to group III. AGS and average global systolic SR showed significant direct correlation with Gensini score. The diagnostic accuracy of 2D STE in prediction of significant LAD stenosis was $92.5 \%$, and it was $89.5 \%$ in prediction of 3 vessels CAD. Conclusion: Strain imaging using 2D STE can predict the territory and severity of CAD with high diagnostic accuracy and can be used as a simple noninvasive diagnostic tool to identify high risk CAD patients.
\end{abstract}

\section{Keywords}

Coronary Artery Disease, Left Ventricle, Strain Imaging 


\section{Introduction}

Noninvasive identification of patients with coronary artery disease (CAD) remains a clinical challenge despite the widespread use, and possible overuse, of imaging and provocative testing; more than $50 \%$ of patients currently referred to coronary angiography show normal or non-obstructive CAD [1].

Recently, the 2-dimensional Speckle Tracking Echocardiography (STE) and its derivatives, including strain and strain rate imaging have been gained substantial clinical interest. Left ventricular longitudinal strain, derived using two-dimensional speckle-tracking echocardiography, has emerged as a noninvasive marker of both global and regional LV dysfunction in patients at risk for developing CAD [2] [3].

Current evidence supports the use of GLS in the detection of moderate to severe obstructive CAD in symptomatic patients. GLS may complement existing diagnostic algorithms and act as an early adjunctive marker of cardiac ischemia [4].

We aimed to assess whether the diagnostic accuracy of 2D-STE global and segmental longitudinal strain (LS) measured at rest may compare to the parameters which can be measured today during conventional Echo. to predict angiographically obstructive CAD.

\section{Material and Methods}

\subsection{Patient Population}

This is a cross-sectional randomized study including a total of 100 patients admitted to Menoufia university hospitals. Patients were enrolled in the study after obtaining their written informed consent, and approval of the local ethics committee of the hospital.

Patients who included in the study were divided according to their presentation into 3 groups; Group I ( $\mathrm{n}=40$ patients): patients presented with ST segment elevation myocardial infarction (STEMI), Group II ( $\mathrm{n}=40$ patients): patients presented with non-ST segment elevation myocardial infarction (NSTEMI) or unstable angina (UA) and Group III ( $\mathrm{n}=20$ patients): patients whose coronary angiography revealed normal vessels, they were referred for catheterization laboratory for suspected CAD with one or more of: typical ischemic chest pain, electrocardiographic (ECG) changes suggesting CAD, positive exercise stress test, positive myocardial perfusion imaging or echocardiographic regional wall motion abnormality suggesting CAD.

\subsection{Exclusion Criteria}

Patients with, congestive heart failure, valvular heart disease, left ventricular ejection fraction less than $50 \%$, interventricular conduction disturbances, very high risk NSTE-ACS and arrhythmias were excluded from our study.

After exclusion of non-responders, drop out participants and those with exclusion criteria, 100 patients completed the study (this number was considered suitable enough sample for statistical analysis with significant results and correlations). 


\subsection{Methods}

All patients were subjected to detailed history, including CAD risk factors, physical examination, Electrocardiography (ECG) and laboratory investigations included, complete blood picture, liver and kidney function. Some patients-who did not have history of CAD-performed a stress imaging test to diagnose CAD.

Echocardiographic images were obtained in the parasternal long-axis and short-axis and apical two-chamber and four-chamber views using standard transducer positions. Vivid 9, General Electric Healthcare (GE Vingmed, Norway) equipped with a harmonic M5S variable-frequency $(1.7-4 \mathrm{MHz})$ phased-array transducer was used. LV dimensions and wall thickness, EF, and left atrial diameter and volume were measured in accordance with the recommendations of the American Society of Echocardiography [5].

Peak early (E) and late (A) transmitral filling velocities were measured from mitral inflow velocities. Early diastolic (e') velocity was obtained by placing a tissue Doppler sample volume at the septal and lateral mitral annulus in the apical four-chamber view, and the mean value was obtained. The E/e' ratio was also calculated.

Blood samples were collected before cardiac catheterization. Patients fasted for $>12$ hours before cardiac catheterization. Blood was collected either from the antecubital vein or indwelling catheter into two $3.2 \%$ trisodium citrate tube after discarding the initial $3 \mathrm{ml}$ of blood. Serum was separated by centrifugation at $2000 \mathrm{~g}$ for 15 minutes and stored at $-70^{\circ} \mathrm{C}$. $\mathrm{CBC}$, liver and renal function were measured.

2D strain analysis was performed offline using the Echopac software (General Electric version 1.8.1.X-Vingmed). All strain images were obtained at a frame rate of $59-82$ frame/s. Three consecutive cardiac cycles were acquired at end expiration breath holding and digitally stored on a hard disk for off-line analysis. In order to measure the timing of cardiac events, LV inflow (mitral) and outflow (aortic) velocities were recorded using Pulsed wave Doppler. Longitudinal strain and strain rate were assessed in the $6 \mathrm{LV}$ walls and the software algorithm automatically segmented the LV into an equidistant segment model in a "bull's eye" plot and each segment was individually analyzed.

The average value of peak longitudinal strain (Esys), the average value of peak systolic strain rate (SRs), peak early diastolic strain rate (SRe), and peak late diastolic strain rate $(\mathrm{SRa})$ were measured at each segment (basal, mid and apical) then the global LV strain and the global LV systolic and diastolic SR were obtained from averaging the peak values of $18 \mathrm{LV}$ segments and used for comparison between the studied groups.

Right and left coronary angiography was performed using multiple projections and analysis was done by professional interventionists who were blind to the cases. Angiographic CAD is defined as $\geq 50 \%$ luminal diameter stenosis of at least one major epicardial coronary artery.

Assessment of the severity of CAD for each coronary angiogram was done 
with the Gensini scoring system a method that assigns a different severity score depending on the degree of luminal narrowing and geographical importance of its lesions. The narrowing of the coronary artery lumen is rated 1 for $1 \%-25 \%$ narrowing, 2 for $26 \%$ - 50\% narrowing, 4 for 51\% - 75\% narrowing, 8 for 76\% $90 \%$ narrowing, 16 for $91 \%$ - 99\% narrowing, 32 for a completely occluded artery. The score is then multiplied by a factor according to the importance of the coronary artery as follows: 5 for Left main (LM); 2.5 for Proximal left anterior descending (LAD) and proximal left circumflex (LCX); 1.5 for mid LAD, 1 for distal LAD, distal LCX and right coronary (RCA) lesions; 0.5 for any other branches (Figure 1). Also severity of CAD was assessed using coronary vessel score in which severity was assessed as no vessel disease, one vessel disease, two vessels disease and three vessels disease.

For STEMI patients, they underwent either rescue PCI after failed thrombolytic therapy or routine PCI strategy after successful reperfusion. Meanwhile for NSTE-ACS very high risk patients were excluded and coronary angiography was done for those who need intervention during the first 24 or 72 hours.

\subsection{Statistical Analysis}

Statistical analysis was performed using Statistical Package for The Social Sciences Version 22 (IBM Corp., Armonk, NY, USA). Quantitative data are expressed as means and standard deviations.

$\mathrm{P}$-Value $\leq 0.05$ was considered to indicate significance. Correlation analysis assesses the strength of association between two variables. Multiple logistic regression analysis was used to detect the associations between lipid profile and both maternal and neonatal complications.

\section{Results}

The patients were classified into 3 groups according to presentation into group I, 40 patients with STEMI and group II, 40 patients with NSTE-ACS and 20 patients represents control group III.

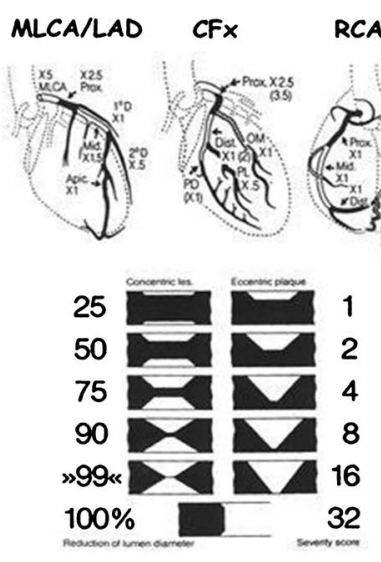

Example: Patient no. 28 (3-VD)

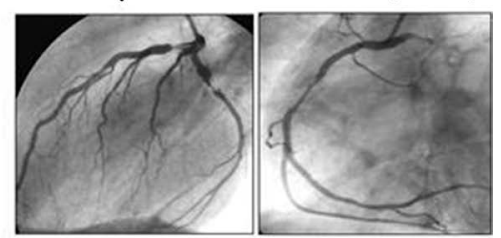

Left $C A$

Right CA

LAD: 75\% concentric (4), proximal (2.5). Score: $4 \times 2.5=10$ LCX: $90 \%$ eccentric (8), proximal (2.5). Score: $8 \times 2.5=20$ RCA: $75 \%$ eccentric (4), middle (1). Score: $4 \times 1=4$

Final Gensini score: $10+20+4=34$

Figure 1. Schematic drawing of the Gensini score. (MLCA, Main left coronary artery; LAD, Left anterior descending; CFX, Circumflex; RCA, Right coronary artery). 
There was a non-significant difference between the studied groups regarding to demographic data (age, gender) and risk factors of CAD (Table 1).

There was a significant difference between group II and control regarding LVESD with $\mathrm{p}$ value 0.008 , there was also a significant difference between Group I, II and III regarding EF\% with p values $0.019,<0.001$ and 0.011 respectively. Regarding FS\%, there was a significant difference between Group I, II and III with $\mathrm{p}$ values $0.018,<0.001$ and 0.013 respectively, with no significant difference regarding other parameters (Table 2 ).

Table 1. Demographic data and risk factors among the studied groups.

\begin{tabular}{ccccccc}
\hline \multirow{2}{*}{ Demographic data } & $\begin{array}{c}\text { Group I } \\
(\mathbf{n}=\mathbf{4 0})\end{array}$ & $\begin{array}{c}\text { Group II } \\
(\mathbf{n}=40)\end{array}$ & $\begin{array}{c}\text { Control } \\
(\mathbf{n}=20)\end{array}$ & \multicolumn{3}{c}{ Test of significance } \\
\cline { 6 - 8 } & & & P1 & P2 & P3 \\
\hline Age (years): Mean \pm SD & $52.20 \pm 11.83$ & $51.97 \pm 14.53$ & $52.75 \pm 10.75$ & 0.862 & 0.834 & 0.94 \\
Sex & & & & & & \\
Male & $31(77.5 \%)$ & $33(82.5 \%)$ & $14(70 \%)$ & 0.527 & 0.268 & 0.576 \\
Female & $9(22.5 \%)$ & $7(17.5 \%)$ & $6(30 \%)$ & & & \\
Smokers & $23(57.5 \%)$ & $26(65 \%)$ & $10(50 \%)$ & 0.582 & 0.264 & 0.491 \\
DM & $11(27.5 \%)$ & $12(30 \%)$ & $6(30 \%)$ & 0.839 & 1 & 0.805 \\
HTN & $13(32.5 \%)$ & $14(35 \%)$ & $5(25 \%)$ & 0.55 & 0.432 & 0.813 \\
Dyslipidemia & $21(52.5 \%)$ & $18(45 \%)$ & $10(50 \%)$ & 0.855 & 0.714 & 0.502 \\
Family history & $18(45 \%)$ & $14(35 \%)$ & $7(35 \%)$ & 0.459 & 1 & 0.361 \\
\hline
\end{tabular}

P1: $\mathrm{p}$ value between Group I and controls; P2: p value between Group II and controls; P3: p value between Group I and II.

Table 2. Conventional Echo among the studied groups.

\begin{tabular}{|c|c|c|c|c|c|c|}
\hline \multirow{2}{*}{ Conventional Echo } & \multirow{2}{*}{$\begin{array}{l}\text { Group I } \\
(\mathrm{n}=40)\end{array}$} & \multirow{2}{*}{$\begin{array}{l}\text { Group II } \\
(\mathrm{n}=40)\end{array}$} & \multirow{2}{*}{$\begin{array}{l}\text { Control } \\
(\mathrm{n}=20)\end{array}$} & \multicolumn{3}{|c|}{ Test of significance } \\
\hline & & & & $\mathrm{P} 1$ & $\mathrm{P} 2$ & P3 \\
\hline $\mathrm{AO} \mathrm{cm}$ & $3.38 \pm 0.29$ & $3.43 \pm 0.44$ & $3.53 \pm 0.38$ & 0.098 & 0.398 & 0.538 \\
\hline LA diameter & $3.97 \pm 0.45$ & $3.88 \pm 0.43$ & $3.91 \pm 0.42$ & 0.594 & 0.817 & 0.355 \\
\hline LVEDD & $5.11 \pm 0.69$ & $5.25 \pm 0.71$ & $5.07 \pm 0.63$ & 0.819 & 0.338 & 0.377 \\
\hline LVESD & $3.66 \pm 0.64$ & $3.94 \pm 0.74$ & $3.44 \pm 0.48$ & 0.173 & $0.008^{*}$ & 0.076 \\
\hline $\mathrm{EF} \%$ & $54.42 \pm 8.93$ & $49.00 \pm 9.74$ & $59.55 \pm 4.27$ & $0.019^{*}$ & $<0.001^{\star}$ & $0.011^{\star}$ \\
\hline FS\% & $28.55 \pm 5.68$ & $25.22 \pm 6.07$ & $31.85 \pm 2.83$ & $0.018^{*}$ & $<0.001^{*}$ & $0.013^{*}$ \\
\hline septum & $0.98 \pm 0.16$ & $1.04 \pm 0.17$ & $1.05 \pm 0.17$ & 0.125 & 0.751 & 0.144 \\
\hline posterior wall & $1.06 \pm 0.14$ & $1.06 \pm 0.17$ & $1.14 \pm 0.12$ & $0.040^{*}$ & 0.09 & 0.888 \\
\hline $\mathrm{E} \mathrm{m} / \mathrm{s}$ & $0.57 \pm 0.18$ & $0.56 \pm 0.14$ & $0.54 \pm 0.13$ & 0.515 & 0.675 & 0.706 \\
\hline $\mathrm{A} \mathrm{m} / \mathrm{s}$ & $0.71 \pm 0.21$ & $0.74 \pm 0.200$ & $0.75 \pm 0.19$ & 0.471 & 0.827 & 0.529 \\
\hline $\mathrm{E} / \mathrm{A}$ ratio & $0.90 \pm 0.51$ & $0.83 \pm 0.46$ & $0.82 \pm 0.50$ & 0.562 & 0.899 & 0.556 \\
\hline
\end{tabular}

P1: p value between Group I and controls; P2: p value between Group II and controls; P3: p value between Group I and II. 
There was a non-significant difference between group I, II and III regarding TAPSE with $\mathrm{p}$ values $0.504,0.844$ and 0.635 respectively. There was a high significant difference when comparing group I with controls and group II with controls regarding MAPSE and RWMA index with $\mathrm{p}$ value $<0.001$ for all (Table 3).

On the other hand, there was a significant difference among Group I \& controls and group II \& controls regarding average LV global average systolic strain $\%$ with $\mathrm{p}$ value $<0.001$ for both, meanwhile there was non-significant difference between group I and group II with p value 0.323 (Table 4, Figure 2).

There was also a statistically significant difference for all walls AGS when comparing group I with controls and group II with controls with $\mathrm{p}$ values less than 0.05 for all, with non-significant values when comparing group I with group II with $\mathrm{p}$ values more than 0.05 except for inferior wall AGS with $\mathrm{p}$ value 0.043 (Table 4, Figure 2).

Regarding cumulative peak systolic strain rate (SRs), it was significant when comparing group I with controls and group II with controls with $p$ value less than 0.001 for both. For cum. SRe \& SRa, it was significant when comparing group I with controls and group II with controls with $\mathrm{p}$ value less than 0.001 (Table 5).

As regarding angiographic finding of the patients groups in detection of diseased

Table 3. TAPSE, MAPSE and RWMA among the studied groups.

\begin{tabular}{ccccccc}
\hline Variables & $\begin{array}{c}\text { Group I } \\
(\mathrm{n}=40)\end{array}$ & $\begin{array}{c}\text { Group II } \\
(\mathrm{n}=40)\end{array}$ & $\begin{array}{c}\text { Control } \\
(\mathrm{n}=20)\end{array}$ & \multicolumn{2}{c}{ Test of significance } \\
\cline { 5 - 7 } & $2.32 \pm 0.25$ & $2.29 \pm 0.30$ & $2.27 \pm 0.20$ & 0.501 & 0.844 & 0.635 \\
TAPSE cm & $1.52 \pm 0.16$ & $1.46 \pm 0.17$ & $2.10 \pm 0.33$ & $<0.001^{*}$ & $<0.001^{*}$ & 0.135 \\
MAPSE cm & $10(0-16)$ & $8.0(0-13)$ & $0(0-0)$ & $<0.001^{*}$ & $<0.001^{*}$ & 0.111 \\
\hline
\end{tabular}

P1: p value between Group I and controls; P2: p value between Group II and controls; P3: p value between Group I and II.

Table 4. LV strain and strain rate among the studied groups.

\begin{tabular}{ccccccc}
\hline LV strain & $\begin{array}{c}\text { Group I } \\
(\mathrm{n}=40)\end{array}$ & $\begin{array}{c}\text { Group II } \\
(\mathrm{n}=40)\end{array}$ & $\begin{array}{c}\text { Control } \\
(\mathrm{n}=20)\end{array}$ & Pest of significance \\
\cline { 5 - 7 } & $-14.11 \pm 5.00$ & $-12.77 \pm 5.61$ & $-22.32 \pm 1.05$ & $<0.001^{*}<0.001^{*}$ & 0.263 \\
\hline Cum. Septal SS & $-14.05 \pm 7.35$ & $-14.67 \pm 6.43$ & $-18.27 \pm 1.03$ & $0.014^{*}$ & $0.016^{*}$ & 0.691 \\
Cum. Lateral SS & $-15.15 \pm 5.55$ & $-13.45 \pm 7.39$ & $-20.47 \pm 0.86$ & $<0.001^{*}<0.001^{*}$ & 0.248 \\
Cum. Anterior SS & $-16.19 \pm 5.31$ & $-13.75 \pm 5.28$ & $-23.18 \pm 1.63$ & $<0.001^{*}<0.001^{*}$ & $0.043^{*}$ \\
Cum. Inferior SS & $-13.30 \pm 6.85$ & $-13.15 \pm 6.74$ & $-16.6 \pm 1.66$ & $<0.001^{*}<0.001^{*}$ & 0.922 \\
Cum. Posterior SS & $-13.13 \pm 5.86$ & $-10.66 \pm 6.95$ & $-24.21 \pm 0.82$ & $<0.001^{*}<0.001^{*}$ & 0.091 \\
Cum. Anteroseptal SS & $-14.93 \pm 3.84$ & $-13.84 \pm 5.78$ & $-21.34 \pm 0.99$ & $<0.001^{*}<0.001^{*}$ & 0.323 \\
\hline Avg global systolic strain \%
\end{tabular}

P1: p value between Group I and controls; P2: p value between Group II and controls; P3: p value between Group I and II. 


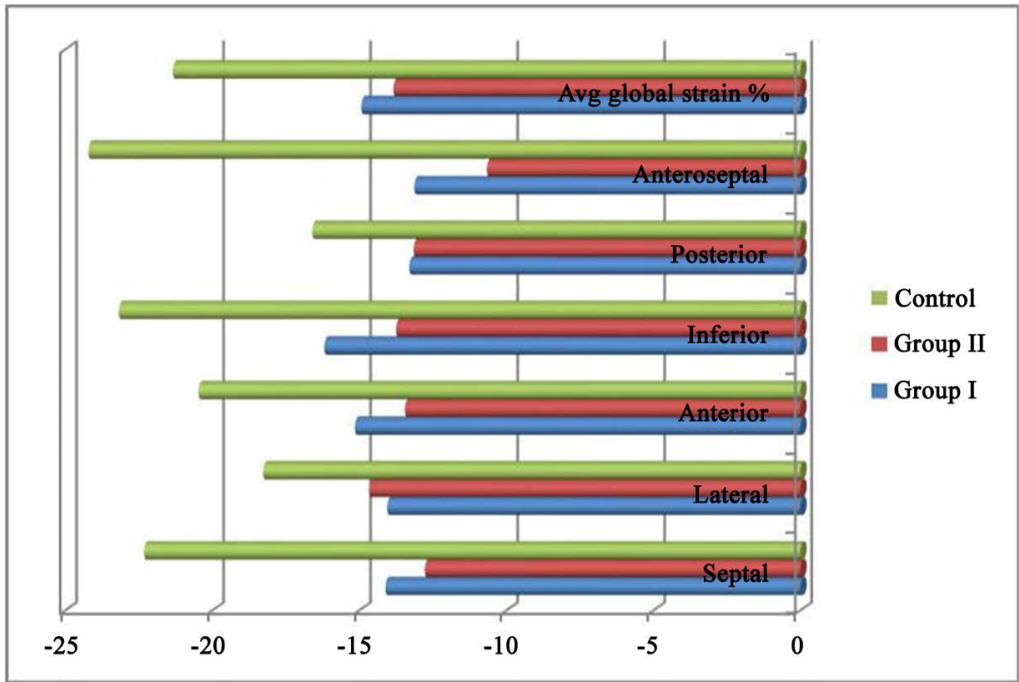

Figure 2. LV strain among the studied groups.

Table 5. Strain rate among the studied groups.

\begin{tabular}{|c|c|c|c|c|c|c|}
\hline \multirow{2}{*}{ Strain rate } & \multirow{2}{*}{$\begin{array}{l}\text { Group I } \\
(\mathrm{n}=40)\end{array}$} & \multirow{2}{*}{$\begin{array}{l}\text { Group II } \\
(\mathrm{n}=40)\end{array}$} & \multirow{2}{*}{$\begin{array}{l}\text { Control } \\
(\mathrm{n}=20)\end{array}$} & \multicolumn{3}{|c|}{ Test of significance } \\
\hline & & & & $\mathrm{P} 1$ & $\mathrm{P} 2$ & P3 \\
\hline \multicolumn{7}{|l|}{ Cum. SRs } \\
\hline Septal & $-0.91 \pm 0.33$ & $-0.83 \pm 0.35$ & $-1.94 \pm 0.11$ & $<0.001^{\star}$ & $<0.001^{\star}$ & 0.313 \\
\hline Lateral & $-0.89 \pm 0.32$ & $-0.95 \pm 0.32$ & $-1.89 \pm 0.14$ & $<0.001^{\star}$ & $<0.001^{\star}$ & 0.425 \\
\hline Anterior & $-0.90 \pm 0.33$ & $-0.83 \pm 0.37$ & $-1.97 \pm 0.17$ & $<0.001^{\star}$ & $<0.001^{\star}$ & 0.372 \\
\hline Inferior & $-1.02 \pm 0.34$ & $-0.95 \pm 0.30$ & $-2.04 \pm 0.12$ & $<0.001^{\star}$ & $<0.001^{\star}$ & 0.368 \\
\hline Posterior & $-0.58 \pm 0.19$ & $-0.67 \pm 0.19$ & $-1.71 \pm 0.13$ & $<0.001^{\star}$ & $<0.001^{\star}$ & $0.043^{*}$ \\
\hline Anteroseptal & $-0.85 \pm 0.34$ & $-0.82 \pm 0.38$ & $-1.87 \pm 0.09$ & $<0.001^{\star}$ & $<0.001^{*}$ & 0.745 \\
\hline Global LV SRs & $-0.86 \pm 0.21$ & $-0.84 \pm 0.22$ & $-1.90 \pm 0.07$ & $<0.001^{\star}$ & $<0.001^{*}$ & 0.752 \\
\hline \multicolumn{7}{|l|}{ Cum. SRe } \\
\hline Septal & $1.07 \pm 0.34$ & $0.89 \pm 0.36$ & $1.97 \pm 0.15$ & $<0.001^{\star}$ & $<0.001^{*}$ & $0.026^{*}$ \\
\hline Lateral & $1.08 \pm 0.37$ & $1.03 \pm 0.40$ & $1.91 \pm 0.15$ & $<0.001^{\star}$ & $<0.001^{\star}$ & 0.517 \\
\hline Anterior & $2.23 \pm 5.20$ & $1.23 \pm 3.28$ & $1.98 \pm 0.17$ & $<0.001^{\star}$ & 0.315 & 0.31 \\
\hline Inferior & $1.09 \pm 0.39$ & $0.94 \pm 0.34$ & $1.99 \pm 0.10$ & $<0.001^{\star}$ & $<0.001^{\star}$ & 0.07 \\
\hline Posterior & $0.99 \pm 0.34$ & $1.07 \pm 0.41$ & $1.73 \pm 0.12$ & $<0.001^{\star}$ & $<0.001^{\star}$ & 0.356 \\
\hline Anteroseptal & $0.96 \pm 0.34$ & $0.95 \pm 0.36$ & $1.90 \pm 0.10$ & $<0.001^{\star}$ & $<0.001^{\star}$ & 0.877 \\
\hline Global LV SRe & $1.24 \pm 0.94$ & $1.02 \pm 0.59$ & $1.91 \pm 0.05$ & $0.002^{*}$ & $<0.001^{\star}$ & 0.218 \\
\hline \multicolumn{7}{|l|}{ Cum. SRa } \\
\hline Septal & $0.73 \pm 0.30$ & $0.61 \pm 0.24$ & $1.98 \pm 0.17$ & $<0.001^{\star}$ & $<0.001^{\star}$ & $0.050^{*}$ \\
\hline Lateral & $0.96 \pm 0.32$ & $0.83 \pm 0.28$ & $1.91 \pm 0.12$ & $<0.001^{\star}$ & $<0.001^{\star}$ & 0.052 \\
\hline Anterior & $1.07 \pm 0.36$ & $0.89 \pm 0.33$ & $2.01 \pm 0.21$ & $<0.001^{\star}$ & $<0.001^{\star}$ & $0.032^{*}$ \\
\hline Inferior & $1.05 \pm 0.33$ & $0.90 \pm 0.33$ & $1.99 \pm 0.13$ & $<0.001^{\star}$ & $<0.001^{\star}$ & $0.038^{*}$ \\
\hline Posterior & $0.96 \pm 0.34$ & $0.93 \pm 0.33$ & $1.77 \pm 0.12$ & $<0.001^{\star}$ & $<0.001^{\star}$ & 0.715 \\
\hline Anteroseptal & $1.00 \pm 0.34$ & $0.88 \pm 0.38$ & $1.88 \pm 0.13$ & $<0.001^{\star}$ & $<0.001^{\star}$ & 0.145 \\
\hline Global LV SRa & $0.96 \pm 0.26$ & $0.84 \pm 0.22$ & $1.92 \pm 0.06$ & $<0.001^{\star}$ & $<0.001^{\star}$ & $0.027^{*}$ \\
\hline
\end{tabular}

P1: p value between Group I and controls; P2: p value between Group II and controls; P3: p value between Group I and II. 
artery, there was $87.5 \%$ with LAD stenosis, $65 \%$ with LCX stenosis and $76.25 \%$ with RCA stenosis and for 2D speckle tracking finding of the patients group in detection of diseased artery, there was 83.75 with LAD stenosis, 62.5 with LCX stenosis and 73.75 with RCA stenosis (Figure 3 ).

Regarding the validity of $2 \mathrm{D}$ speckle tracking in detection of diseased coronary artery, it was good predictor for LAD stenosis with $90 \%$ sensitivity and $60 \%$ specificity, for LCX stenosis $86.5 \%$ sensitivity and $82.1 \%$ specificity and finally for RCA stenosis $88.5 \%$ sensitivity and $73.7 \%$ specificity (Table 6 ). Also, Figure 7 shows segmental longitudinal strain and strain rate in patient with subtotal proximal LAD occlusion, there is reduced regional strain and strain rate in the distribution of diseased LAD.

Regarding the number of stenotic vessels detected by coronary angiography for patients' groups, $18.75 \%$ patients had one vessel disease, $28.75 \%$ patients had two vessels disease and $52.5 \%$ had three-vessel disease and for suspected by $2 \mathrm{D}$ speckle tracking, $20 \%$ patients had one vessel disease, $32.5 \%$ patients had two vessels disease and $47.5 \%$ had three-vessel disease (Figure 4 ).

Regarding the validity of 2D speckle tracking in detection of number of stenotic coronary arteries, it was good predictor for three vessels disease with $88 \%$ sensitivity and $78.9 \%$ specificity, for two vessels disease $86.9 \%$ sensitivity and $71.7 \%$ specificity and finally for one vessel disease stenosis $80 \%$ sensitivity and 91.8\% specificity (Table 7 ).

The best cut-off value considering AGS\% in prediction of severity of coronary artery disease based on Gensini score is -11 with $93.3 \%$ sensitivity and $98.4 \%$ specificity, for SRs is -0.67 with $86.7 \%$ sensitivity and $89.2 \%$ specificity, for SRe is 0.89 with $86.7 \%$ sensitivity and $72.3 \%$ specificity, finally for SRa is 0.85 with 93.3\% sensitivity and $69.2 \%$ specificity (Table 8 , Figure 5 ).

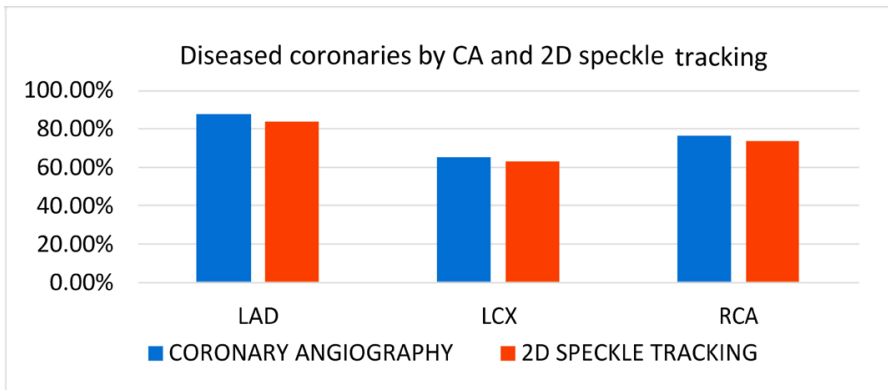

Figure 3. Number of patients with significant stenosis in each coronary artery as detected by CA and as suspected by 2 D STE.

Table 6. Diagnostic accuracy of 2D speckle tracking in detection of diseased coronary artery.

\begin{tabular}{cccccc}
\hline Variable & Sensitivity & Specificity & Accuracy & PPV & NPV \\
\hline LAD & $90 \%$ & $60 \%$ & $92.50 \%$ & $94 \%$ & $46.20 \%$ \\
LCX & $86.50 \%$ & $82.10 \%$ & $71.25 \%$ & $90 \%$ & $76.60 \%$ \\
RCA & $88.50 \%$ & $73.70 \%$ & $82.50 \%$ & $91.50 \%$ & $66.70 \%$ \\
\hline
\end{tabular}




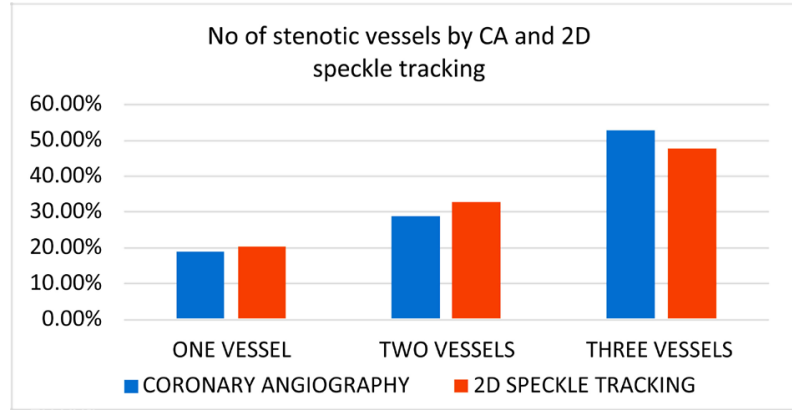

Figure 4. No. of patients with different vessel score as detected by CA and as suspected by 2D STE.

Table 7. Diagnostic accuracy of 2D speckle tracking in detection of number of stenotic coronary arteries.

\begin{tabular}{cccccc}
\hline Variable & Sensitivity & Specificity & Accuracy & PPV & NPV \\
\hline One vessel & $80 \%$ & $91.80 \%$ & $93.50 \%$ & $79.70 \%$ & $91.70 \%$ \\
Two vessels & $86.90 \%$ & $71.70 \%$ & $82.25 \%$ & $75.80 \%$ & $88.90 \%$ \\
Three vessels & $88 \%$ & $78.90 \%$ & $89.50 \%$ & $94.60 \%$ & $87.50 \%$ \\
\hline
\end{tabular}

Table 8. Accuracy of AGS\% and Strain rate in prediction of severity of CAD.

\begin{tabular}{ccccccccc}
\hline Variables & AUC & $95 \%$ CI & Cutoff & SN & SP & PPV & NPV & Accuracy \\
\hline AGS\% & 0.971 & $0.93-1$ & $<-11$ & $93.30 \%$ & $98.40 \%$ & 93.3 & 98.4 & $97.50 \%$ \\
SRs & 0.898 & $0.82-0.97$ & $<-0.67$ & $86.70 \%$ & $89.20 \%$ & 65 & 96.7 & $88.70 \%$ \\
SRe & 0.832 & $0.74-0.92$ & $>0.89$ & $86.70 \%$ & $72.30 \%$ & 95.9 & 64.5 & $83.70 \%$ \\
SRa & 0.748 & $0.63-0.87$ & $>0.85$ & $93.30 \%$ & $69.20 \%$ & 98.2 & 58.3 & $86.20 \%$ \\
\hline
\end{tabular}

SN: Sensitivity, SP: Specificity, PPV: Positive, Predictive Value, NPV: Negative Predictive Value, AUR: Area Under Receiver Operating Characteristic curve, 95\% CI: 95\% Confidence Interval.

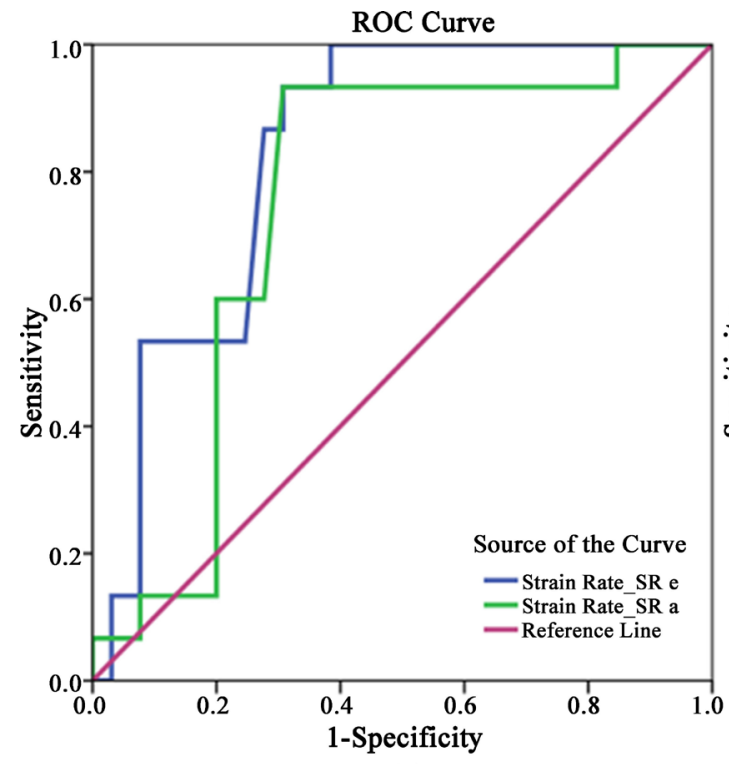

(a)

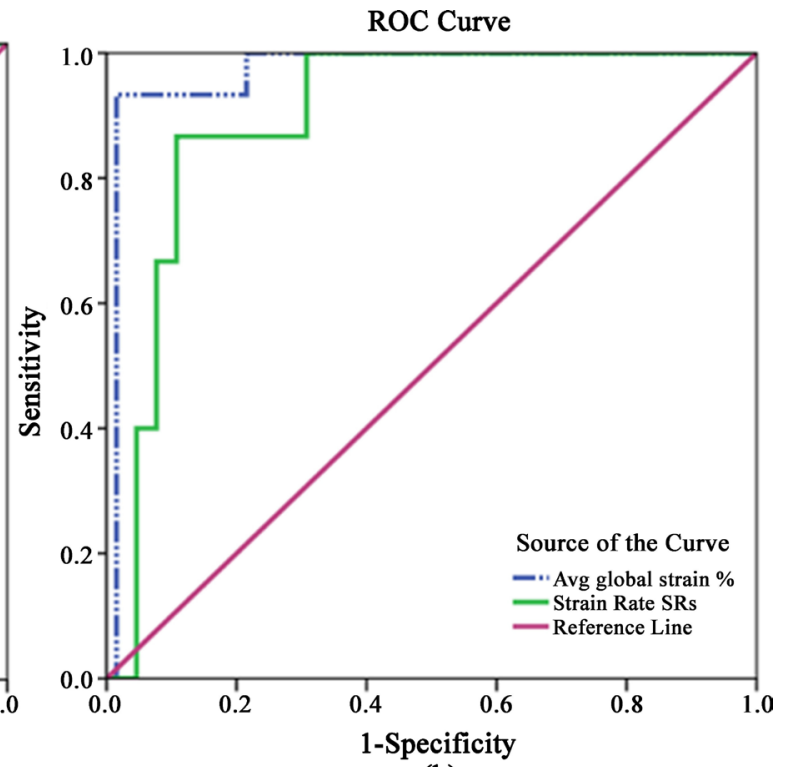

(b)

Figure 5. ROC curve for predication of CAD with AGS\% and SRs (left) \& SRe and SRa (right). 
A multivariate logistic regression model was performed for independent predictors of severity of coronary artery disease based on Gensini score. The result showed that EF\%, FS, TAPSE, MAPSE, average global strain, SRs and SRa are independent predictors for coronary artery disease with $\mathrm{p}$ values $0.001,0.007$, $0.007,0.001,0.006,0.011$ and 0.026 respectively (Table 9 , Figure 6 ). So, the best

Table 9. Multivariate linear regression analysis for independent predictors of severity of coronary artery disease based on Gensini score.

\begin{tabular}{cccc}
\hline Independent predictors & $\mathrm{b}$ & Std. Error & $\mathrm{p}$ \\
\hline LVEDD & 44.37 & 28.9 & 0.13 \\
LVESD & -68.24 & 39.1 & 0.086 \\
EF\% & -12.62 & 3.4 & $0.001^{*}$ \\
FS\% & 14.88 & 5.3 & $0.007^{*}$ \\
TAPSE cm & -22.47 & 8.1 & $0.007^{\star}$ \\
MAPSE cm & -49.53 & 13.5 & $<0.001^{*}$ \\
RWMA & -0.065 & 0.36 & 0.859 \\
Average global strain \% & 1.77 & 0.71 & $0.006^{*}$ \\
Strain rate SRs & 42.37 & 16.1 & $0.011^{*}$ \\
Strain rate SRe & -0.07 & 2.6 & 0.979 \\
Strain rate SRa & -25.59 & 11.1 & $0.026^{*}$ \\
\hline
\end{tabular}
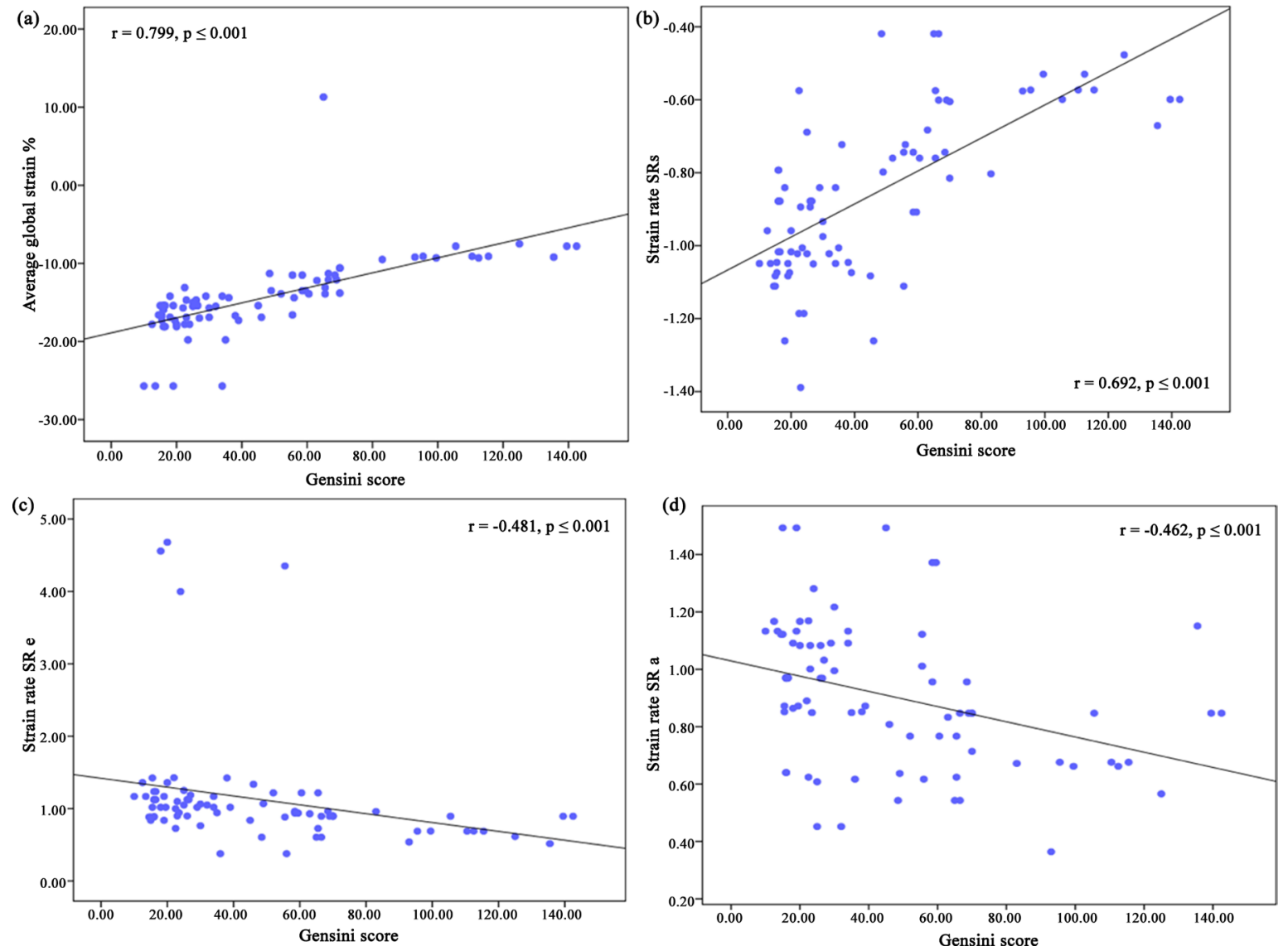

Figure 6. Scatter diagram for correlation between Gensini score and AGS, SRs, SRe and SRa. 


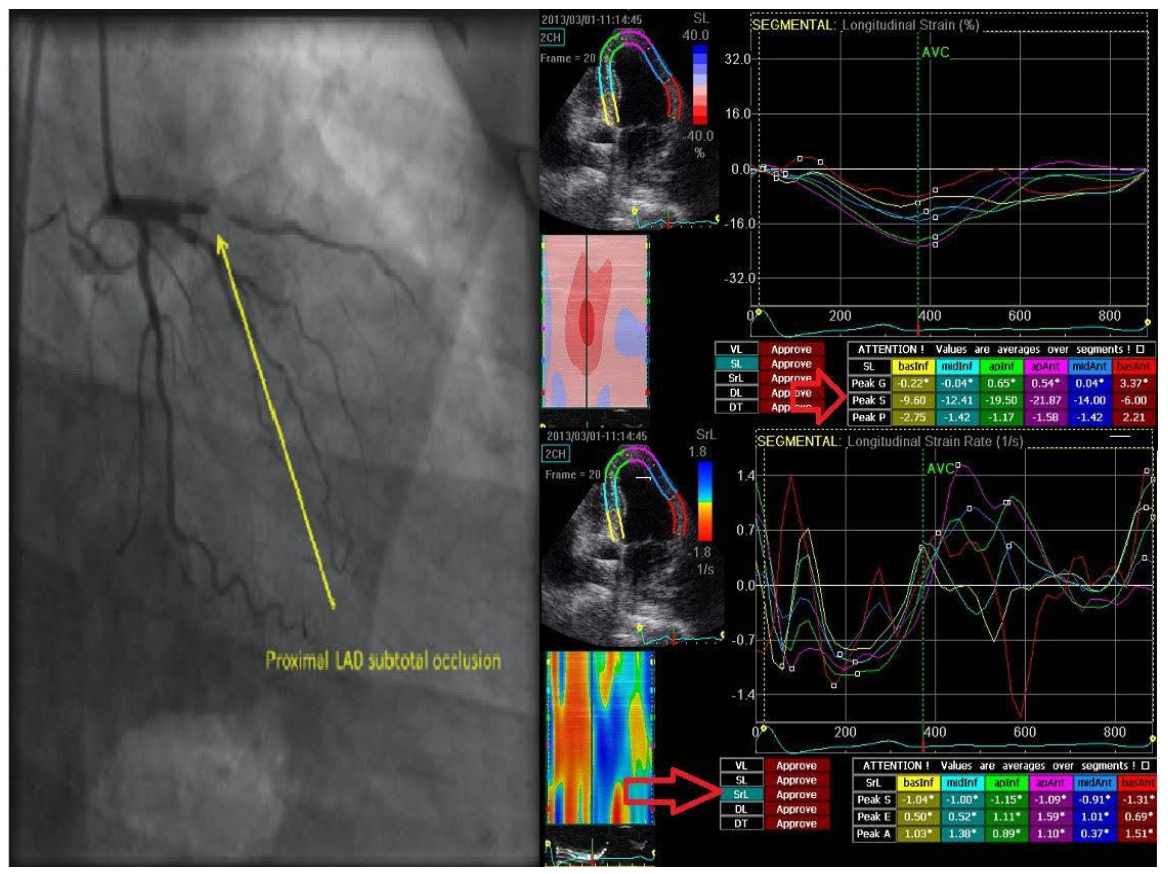

Figure 7. Segmental longitudinal strain and strain rate in patient with subtotal proximal LAD occlusion, there is reduced regional strain and strain rate in the distribution of diseased LAD.

diagnostic accuracy in prediction of severity of coronary artery disease was by AGS\% (97.5\%) followed by SRs (88.7\%) then SRa (86.2\%) and finally SRe (83.7\%).

\section{Discussion}

Noninvasive identification of patients with coronary artery disease (CAD) remains a clinical challenge despite the widespread use, and possible overuse, of imaging and provocative testing; more than $50 \%$ of patients currently referred to coronary angiography show normal or non-obstructive CAD [1].

The 2-dimensional Speckle Tracking Echocardiography (STE) and its derivatives, including strain and strain rate imaging have been gained substantial clinical interest to surmount those limitations [3].

We enrolled 100 patients in our study with suspected coronary artery disease, STE using AGS was done to those patients followed by coronary angiography.

Our results found no statistically significant difference between the three groups as regarding demographic data and risk factors. This was contrary to Conte L. et al. [6] who measured the degree of longitudinal fibers dysfunction by STE in diabetic and obese patients and found reduction in GLPS in those patients. Also, Rostamzadeh et al. stated that of conventional risk factors, diabetes mellitus (55\%) and hypertension (67\%) were more frequent in the high-risk group when compared with low-risk and normal groups [1].

We found that significant difference was present between the three groups as regard LVESD, FS and EF\% ( $\mathrm{p}$ value < 0.05). Şahin et al. agreed with us and 
demonstrated that EF\% have significant difference present between the two groups of acute MI with preserved coronary flow and Impaired coronary flow in 880 consecutive patients with STEMI undergoing primary PCI [7].

Our results found that there was a statistically significant correlation between decreased values of the AGS and presence of obstructive CAD by coronary angiography with a cutoff value of -11 (provided that there is associated decrease in the longitudinal strain of group of segments "territory"), and the cut off value for SRs was -0.67 , for SRe was 0.89 and for SRa was 0.85 . There was also a statistically significant difference was found among the group I and group II regarding affecting wall Cum. Systolic Strain (P-value $<0.001)$.

This finding was consistent with the hypothesis of Radwan and Hussein who stated that longitudinal myocardial deformation has a good predictive value for diagnosis of obstructive CAD [8].

Recent researches also described that patients who had normal resting LV function using $2 \mathrm{D}$ echocardiography and proved to have severe CAD angiographically, those could be discovered by presence of decreased AGS values in STE [9] [10].

Also, Huang et al. demonstrated that changes in AGS of LV aid in early diagnosis of high-risk patients for coronary atherosclerotic disease [11].

We found also that AGS, SRe and SRa values had a strong correlation with coronary artery disease severity by Gensini score, so it is considered as a good test to rule out presence of obstructive CAD, thus we can mostly avoid coronary angiography for patients with normal AGS.

Results by Rostamzadeh et al. agreed with our results and stated that GLS and GLSR of left ventricle were significantly lower in the high-risk group ( $p=0.047$ and $p=0.004$ respectively). Receiver operating characteristics curve showed that the optimal values of GLS and GLSR, for detecting the severe CAD were $-17 \%$ and $-1 \mathrm{~s}^{-1}$ with the sensitivities of $77 \%$ and $71 \%$ and the specificities of $63 \%$ and $67 \%$ respectively [1].

They also stated that the GLSR was also significantly lower in the high-risk group when compared with the low-risk and normal groups $(-0.9 \pm 0.2,-1 \pm 0.2$ and $-1.1 \pm 0.2 \mathrm{~s}^{-1}$, respectively, $p=0.004$ ), in agreement with our data [1].

Tanaka et al. stated that the SRe was significantly correlated with SRs in all studied segments $(r=0.55, p<0.0001)$. The average values of SR indices in each patient were defined as SSR index and ESR index, which were derived by dividing the summed value of the SRs or SRe by the number of respective assessable segments. A significant positive relationship was observed between the SRs index and left ventricular ejection fraction $(r=0.85, p<0.0001)$ [12].

We also found that reduced global systolic strain associated with reduced strain in group of segments (territory), are significantly correlated to coronary artery lesions discovered by coronary angiography.

This was in agreement with Fahim et al. who predicted the extent and site of lesions in coronary arteries in patients suspected to have stable CAD depending 
on values of segmental strain [13].

These results were supported by Vrettos et al., who correlated the global longitudinal peak systolic strain with the site and severity of coronary artery lesions assessed by coronary angiography [14].

This relation between segmental longitudinal strain values reduction by STE and site of coronary artery lesion by coronary angiography is also declared by Moustafa et al. [15].

While, Montgomery et al., declared that abnormal myocardial deformation at rest identifies significant CAD especially for LAD stenosis [16].

Also, Raja, established that systolic strain has the highest specificity for detection of obstructive CAD in LAD followed by RCA and LCX [17].

\section{Limitations}

The study lacked a large validation population. Further prospective studies are thus needed to confirm our results. Also, the definition of coronary arteries disease was based on angiographic views of 2D X-ray, we did not use IVUS or FFR which may interfere with the decision of the interpretation of coronary angiography.

\section{Conclusion}

Strain imaging using 2D STE can predict the territory and severity of CAD with high diagnostic accuracy and can be used as a simple noninvasive diagnostic tool to identify high risk CAD patients.

\section{Conflicts of Interest}

The authors declare no conflicts of interest regarding the publication of this paper.

\section{References}

[1] Rostamzadeh, A., Shojaeifard, M., Rezaei, Y., et al. (2015) Diagnostic Accuracy of Myocardial Deformation Indices for Detecting High Risk Coronary Artery Disease in Patients without Regional Wall Motion Abnormality. International Journal of Experimental Clinical Medicine, 8, 9412.

[2] Liou, K., Negishi, K., Ho, S., Russell, E.A., et al. (2016) Detection of Obstructive Coronary Artery Disease Using Peak Systolic Global Longitudinal Strain Derived by Two-Dimensional Speckle-Tracking: A Systematic Review and Meta-Analysis. Journal of American Society of Echo, 29, 724-735.e4. https://doi.org/10.1016/j.echo.2016.03.002

[3] Eitan, A., Kehat, I., Mutlak, D., et al. (2018) Longitudinal Two-Dimensional Strain for the Diagnosis of Left Ventricular Segmental Dysfunction in Patients with Acute Myocardial Infarction. The International Journal of Cardiovascular Imaging, 34, 237-249. https://doi.org/10.1007/s10554-017-1231-y

[4] Gaibazzi, N., Pigazzani, F., Reverberi, C., et al. (2014) Rest Global Longitudinal 2D Strain to Detect Coronary Artery Disease in Patients Undergoing Stress Echocardiography: A Comparison with Wall-Motion and Coronary Flow Reserve Responses. 
Echo Research Practice, 1, 61 -70. https://doi.org/10.1530/ERP-14-0020

[5] Gottdiener, J.S., Bednarz, J., Devereux, R., et al. (2004) American Society of Echocardiography Recommendations for Use of Echocardiography in Clinical Trials: A Report from the American Society of Echocardiography's Guidelines and Standards Committee and the Task Force on Echocardiography in Clinical Trials. Journal of American Society of Echo, 17, 1086-1119. https://doi.org/10.1016/S0894-7317(04)00675-3

[6] Conte, L., Fabiani, I., Barletta, V., et al. (2013) Early Detection of Left Ventricular Dysfunction in Diabetes Mellitus Patients with Normal Ejection Fraction, Stratified by BMI: A Preliminary Speckle Tracking Echocardiography Study. Journal of Cardiovascular Echography, 23, 73-80. https://doi.org/10.4103/2211-4122.123953

[7] Şahin, D.Y., Gür, M., Elbasan, Z., et al. (2013) Predictors of Preinterventional Patency of Infarct-Related Artery in Patients with ST-Segment Elevation Myocardial Infarction: Importance of Neutrophil to Lymphocyte Ratio and Uric Acid Level. Experimental Clinical Cardiology, 18, e77.

[8] Radwan, H. and Hussein, E. (2017) Value of Global Longitudinal Strain by Two Dimensional Speckle Tracking Echocardiography in Predicting Coronary Artery Disease Severity. The Egyptian Heart Journal, 69, 95-101.

https://doi.org/10.1016/j.ehj.2016.08.001

[9] Moustafa, S., Elrabat, K., Swailem, F., et al. (2017) The Correlation between Speckle Tracking Echocardiography and Coronary Artery Disease in Patients with Suspected Stable Angina Pectoris. Indian Heart Journal, 56, 63-80. https://doi.org/10.1016/j.ihj.2017.09.220

[10] Hubbard, R.T., Arciniegas Calle, M.C., Barros-Gomes, S., et al. (2017) 2-Dimensional Speckle Tracking Echocardiography Predicts Severe Coronary Artery Disease in Women with Normal Left Ventricular Function: A Case-Control Study. BMC Cardiovascular Disorders, 17, 231. https://doi.org/10.1186/s12872-017-0656-5

[11] Huang, K.-W., Liu, H., Bai, G., et al. (2017) Change in Left Ventricular Global Longitudinal Peak Strain for Early Diagnosis of High-Risk Coronary Atherosclerotic Heart Disease in Elderly Patients: Study Protocol for a Single-Center Diagnostic Trial. Clinical Trials in Degenerative Diseases, 18, e77. https://doi.org/10.4103/2542-3975.216583

[12] Tanaka, H., Kawai, H., Tatsumi, K., et al. (2007) Relationship between Regional and Global Left Ventricular Systolic and Diastolic Function in Patients with Coronary Artery Disease Assessed by Strain Rate Imaging. Circulation, 71, 517-523. https://doi.org/10.1253/circj.71.517

[13] Fahim, M.W.G., Bakhoum, S.Y., Abdelmoneim, Y., et al. (2015) Value of Resting Myocardial Deformation Assessment by Two Dimensional Speckle Tracking Echocardiography in Detecting the Presence, and Predicting the Extent and Localization of Coronary Artery Affection in Patients with Suspected Stable Coronary Artery Disease. The Egyptian Heart Journal, 68, 171-179.

https://doi.org/10.1016/j.ehj.2016.02.001

[14] Vrettos, A., Dawson, D., Grigoratos, C., et al. (2016) Correlation between Global Longitudinal Peak Systolic Strain and Coronary Artery Disease Severity as Assessed by the Angiographically Derived SYNTAX Score. Echo Research and Practice, 3, 29-34. https://doi.org/10.1530/ERP-16-0005

[15] Moustafa, S., Elrabat, K., Swailem, F., et al. (2017) The Correlation between Speckle Tracking Echocardiography and Coronary Artery Disease in Patients with Suspected Stable Angina Pectoris. Indian Heart Journal, 24, 45-59. 
[16] Montgomery, D.E., Puthumana, J.J., Fox, J.M., et al. (2012) Global Longitudinal Strain Aids the Detection of Non-Obstructive Coronary Artery Disease in the Resting Echocardiogram. European Heart Journal of Cardiovascular Imaging, 13, 579-587. https://doi.org/10.1093/ejechocard/jer282

[17] Raja, D. and Khanna, R. (2015) Detection and Location of Obstructive Coronary Artery Disease in Patients of Chronic Stable Angina by Strain and Strain Rate (Myocardial Deformation Parameters) in the Resting Echocardiogram. Indian Heart Journal, 67, S88-S89. https://doi.org/10.1016/j.ihj.2015.10.216 